\title{
Memory and display search in binary classification reaction time
}

\author{
PETER HOWELL \\ University College London, Gower Street, London, W. C.1, England \\ and \\ JANET E. STOCKDALE \\ London School of Economics and Political Science, London, England
}

\begin{abstract}
Character classification tasks and "same"."different" judgments of letter strings were examined with reference to major experimental findings and the models proposed to explain them. An experiment is reported which was designed to investigate similarities between these two paradigms. The subject's task was to scan a display and decide whether all the items in the display belonged to a criterion set; the location of the items in the display was irrelevant to the decision. Two classes of model were considered. First, for models in which display encoding time increases as a function of the number of items on display, an exhaustive-memory/exhaustive-display model offered a reasonable explanation of the data. Second, for models in which display encoding time is a constant, examination of the data in terms of the hypothetical number of memory and display comparisons demanded by the task revealed that negative responses were lengthened relative to positive responses. A one-processor model which postulates an exhaustive-memory self-terminating display search followed by a rechecking process on those trials in which a negative outcome ensues satisfactorily explains the latency and serial position data.
\end{abstract}

In a recent authoritative review. Nickerson (1972) divides binary-classification experiments into character classitication and "same"-" different" judgment tasks. The majority of character classification and "same"." different" experiments use reaction time (RT) as the dependent variable but differ with respect to the decision rule the subject must follow: character classification requires a decision as to whether one or more displayed characters. sometimes termed the test set (TS) and usually alphanumeric, are or are not members of a criterion set (CS): "same".-"different" tasks require a decision whether two stimuli are the same or different with respect to a preselected set of attributes. The stimuli in the "same"." different" task are usually nonalphanumeric. but Bamber (1969) has reported an experiment in which the attributes were the individual alphabetic characters making up a string. An attribute can then take one of two values depending on whether the same or a different item occupies a given position in the display. The limiting case of the character classification task is where both the CS and the TS contain a single item. This is, then. logically similar to the limiting case of the

Requests for reprints should be sent to Peter Howell. who is now at the Laboratory of Experimental Psychology, University of Sussex. Brighton BN19QG, England. This research was supported in part by a Science Research Council grant to Professor R. J. Audley. and by a grant to the second author from the Social Research Division of the London School of Economics. Thanks are due to E. J. Hammond for programming on-line control of the experiment and to Professor R. J. Audley, D. J. Caudrey, and Dr. D. J. Powell for comments during the preparation of this paper. "same" -"different" judgments of letter strings [one letter (or attribute) in the CS which is same or different from the one letter (attribute) in the TS].

The hypotheses usually offered to explain the results of these two types of experiment are the four search models resulting from the possible combinations of the two conceptual contrasts serial or parallel and exhaustive or self-terminating. Serial or parallel refers to whether memory representations of characters or attributes are compared with the processed TS one at a time or simultaneously. Exhaustive or self-terminating refers to whether all possible comparisons of the encoded representation of the CS with the processed TS are made, or only as many as are needed to enable a decision to be made.

Character classification experiments have, in the main. employed the Sternberg paradigm. According to this paradigm, a set of elements is selected arbitrarily from a defined stimulus ensemble and constitutes the CS or positive set which is presented as a list for the subject to memorize. The size of the positive set may be varied and defines the memory load (M) imposed on the subject. On each trial, a single TS is presented and the subject's task is to make a positive response if this is a member of the CS. or if it is not then to make a negative response.

Typically, character classification results show that RT increases linearly with $M$ at the same rate for positive and negative responses, with only an intercept difference between the two kinds of response. From the linearity of the functions. Sternberg (1966) has argued for a serial process. and from the equal slopes. 
for an exhaustive scan, since, if the memory scan had been self-terminating. positive trials would have required on average only half the comparisons demanded by negative trials.

In an extension of the basic character classification task, Sternberg (Note 1) presented subjects with from one to four digits to memorize and then presented tachistoscopically a linear array of one, two, or three digits. The subject makes a positive response if the CS and IS have any elements in common and otherwise makes a negative response. The presence of a positive set item is, then, sufficient for a decision to be made. To aid our analysis, we define a decision item as one whose value on the scanned dimension is sufticient to enable classification of that dimension, under optimal search conditions. In the modified character classification task, a positive item acts as a decision item in both memory and display.

For this type of task. Sternberg found that for all TS sizes greater than one, negative RTs increased at a faster rate than positive RTs with increasing length of the $\mathrm{CS}$. He considered that these data demonstrated that, while scanning of the CS is exhaustive for each itcm in the TS, scanning of the TS is self-terminating. This conclusion was supported by a reanalysis of a similar experiment by Nickerson (1966). This interpretation is not without its difficulties. First, no serial position effects (i.e., no dependence of $R T$ on position of the common item in either list) were reported, which may be explained by the additional assumption of a random order of search in the self-terminating model. and. second, quantitative estimates of the slopes in the two experiments reveal that the negative slope is not twice the positive slope but somewhat less than this.

This extension of the paradigm does, however. allow us to develop our analogy between character classitication and "same".."different" judgments of letter strings. Consider first the task which subjects encountered in the Bamber (1969) experiment. Subjects were required to judge whether or not two successively presented horizontal rows of letters, containing equal numbers of letters, were identical (i.e., contained the same letters in the same order). The length (1) of the letter strings was varied from one to four items and the number of different items from one to 1 . As soon as an item, which was not contained in the CS is processed, a decision can be made. In "same"."different" judgments, an item present in the display, but absent from memory, acts as a decision item.

In the Bamber experiment, a plot of RT as a function of 1 revealed a marked upward concavity for same responses, which was interpreted by Bamber as inconsistent with any model which describes the data in terms of a single search process. "Different" judgments were consistent with a self-terminating model, but "same" responses were faster than predicted. This "fast-same" phenomenon has been reported by a number of experimenters who have eniployed multidimensional stimuli in "same""different" tasks (see the review by Nickerson, 1972).

These data led Bamber to propose a model in which two stimulus-comparison processors, one fast and the other slow, are assumed to operate simultaneously. When the CS and TS are the same, the fast processor is able to operate on the basis of the simple identity of physical stinualus characteristics. When the two stimulus sets are different, the fast processor is unable to operate and negative responses must rely on the output of the slow processor. This model therefore explains why the negative RTs are consistent with a serial self-terminating model, but the positive responses are faster than predicted. The model was tested in experiments carried out by Bamber (1972) and Bamber and Paine (1973). Both experiments were designed to eliminate the intervention of the fast processor so that both positive and negative responses would be initiated by the slow processor. For example. Bamber (1972) required subjects to judge whether two letters, which were always physically different. were nominally the same or different. If it is assumed that the last processor is incapable of making nominal identity judgments, then both positive and negative responses should be consistent with a serial self-terninating model. However, positive responses were still faster than predicted, implying that the "fast-same" phenomenon is not merely the product of a physical match. The experinent of Bamber and Paine also failed to dissociate the two processors, and so the results of both experiments cast some doubt on the validity of the model.

Consideration of character classification tasks and "sane".-"different" judgments has led us to devise a task in which the subject searches the TS in order to decide whether or not it contains all the members of the CS, the CS and TS being of the same length. In this task, the nature of a decision item is the presence of an item in the display which is absent from nemory, i.e., the same as in the "same"."different" judgment task. The proposed task deviates from the traditional "same"."different" judgment of letter strings, in that, as in the moditied character classification task, position of the items will be randomized.

In designing our task, an important procedural difference between Sternberg (Note 1) and Bamber (1969) emerges. In the character classification task used by Sternberg (Note 1), there was a maximum of one decision item in the TS, RT being investigated as a function of position of that item. In contrast, the "same"."different" judgment of letter strings in the Bamber (1969) experiment could contain from one to 1 decision items, RT being examined as a function of the number of decision items. However, in the Bamber experiment, the 
number of decision items for a fixed list length is confounded with the position of these items (i.e., as the number of decision items increases, the probability of encountering a decision item in a given position also increases). For this reason, in the present experiment, when the TS differed from the CS. it did so with respect to one item only, position of the decision item being controlled. Stimulus material was chosen so as to avoid possible ordinal position effects, arising from natural sequencing inherent in the stimulus set. Such effects create problems in providing an adequate model for search tasks. For example, there is evidence that search times depend on whether or not items are formed into organized sets (Rosenbaum, 1974).

A major point of interest in the present experiment will be the nature of the functions fitted to the RTs of negative and positive responses. If it is assumed that information in memory is compared with individual displayed items, not in a one (memory) to one (display) fashion, as is assumed by "same"-"different" theorists, but by means of an exhaustive or self-terminating comparison for both memory and display, the relation between $R T$ and $M$ would be expected to be positively accelerating. This would be consistent with the upward concavity reported in Bamber (1969, 1972).

In the experiments of Nickerson (1966) and Stemberg (Note 1). Sternberg observed that there is a common intercept for both positive and negative responses as the number of items on display increases. Another feature of these two experiments is that display and memory comparisons require the same amount of time (Nickerson, 1972). Assuming that display encoding time is a constant, our inclusion of a control condition in which memory load is varied but display load is constant provides a baseline against which to examine the intercepts and slopes of the RT functions for positive and negative responses when both memory and display loads are varied.

This approach permits a stage analysis of the "last-same" phenomenon. Differences in comparison time between positive and negative responses would lead us to conclude that the "fast-same" phenomenon is a feature of the search stage, any other differences that it is a feature of one of the other stages involved in classitication.

\section{METHOD}

\section{Subjects}

Eight subjects, four male and four female, participated in the experiment. Each subject was paid a total of $£ 2$ for the two $11 / 2-h$ sessions. No practice was given, but all of the eight subjects had previously performed in RT experiments.

\section{Stimulus Materials}

The stimulus ensemble comprised the eight letter-like shapes shown in Figure 1. The shapes were constructed from the elements of a 4 by 6 dot matrix, $7 \mathrm{~mm}$ in height and varying in width from

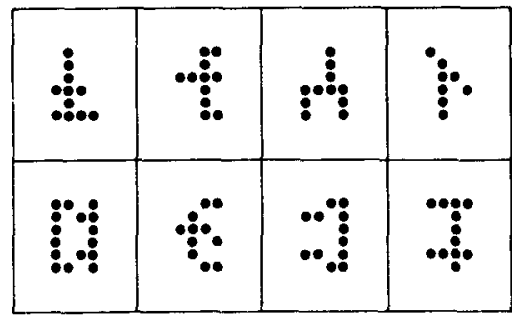

Figure 1. The stimulus ensemble of eight letter-like shapes.

$6 \mathrm{~mm}$ (one character) to $28 \mathrm{~mm}$ (four characters). These were displayed on a $36-\mathrm{cm}$ osciloscope, which the subject viewed from a distance of approximately $60 \mathrm{~cm}$.

\section{Apparatus}

The experiment was run under the control of a PDP-12 computer connected with a Teletype, an oscilloscope, and two pairs of response keys, one set for responding and the other to terminate the CS. Response latencies were measured to an accuracy of $1 \mathrm{msec}$.

\section{Experimental Conditions}

All subjects received two conditions. One condition $(D=1)$ represented a replication of the basic Sternberg characterclassification task in which, following the presentation of a CS of size $M$, a single test item is displayed. Under this condition, the memory load. M. was varied but the display load, D. remained constant at a single item. The subject's task was to decide whether or not the single TS was a member of the CS. Under the other condition $(D=M)$, both the memory and display loads were permitted to vary, but on any given trial the display load was always equal to the memory load. In this case, the subject's task was to decide whether the identity of the items in the TS was the same as those in the CS.

Within each condition, memory loads of $1,2,3$, and 4 were used. Under each of the two experimental conditions, subjects received 96 trials at each of the four memory loads, and of these $50 \%$ required a positive and $50 \%$ a negative response.

\section{Design}

Each subject participated in two experimental sessions. The order of the two conditions, $D=1$ and $D=M$, was counterbalanced across the two sessions. Trials with the same memory load (M $=1,2,3$, and 4$)$ were all grouped together in a block, and the order of presentation of the four blocks of trials within an experimental session were determined by reference to a Latin square in the following way. Each of the four subjects performing condition $D=1$ in the first session was allocated to one order of a Latin square which detined, within the first experimental session. the order of presentation of the four blocks of trials representing the four different memory loads. This square was replicated across the four subjects performing condition $D=M$ in the first session. In the second session, subjects were assigned to different orders within the Latin square with the constraint that all orders were represented. Four subjects made a positive response with their right hands, four subjects with their left hands, and this was counterbalanced across order of conditions.

\section{Stimulus Sequences}

For the $D=1$ condition, a population of $M+4$ stimulus elements was detined prior to a block of trials with a given value of $M$. On any trial, the CS was randomly selected from this ensemble and presented as a horizontal display on the computer oscilloscope. The subject was instructed that he could look at this set of stimuli as long as he wished. In order to terminate the memory display and to commence the trial, he was to use his thumbs to depress two keys which were used for no other purpose in the experiment. One second later, the single TS was displayed. The subject was 
instructed to decide whether or not the TS was a member of the CS and to respond as quickly and accurately as possible by depressing one of two keys, on which he rested his index fingers, one key signaling a positive and the other a negative response.

For the $D=M$ condition, a similar procedure was adopted, except that the TS now contained the same number of stimulus items as displayed in the CS. The test items were displayed in random order. and the subject's task was to decide whether the identity of the items in the TS was the same as those in the CS. The location of the items was irrelevant to this decision. A negative trial was defined by the presence of a single negative set item, i.e., an item not previously displayed in the CS, and the frequency with which a negative item appeared at each serial position was equated over the sequence of 96 trials within each memory load. One second after the subject's response a new CS was presented and the sequence of events was repeated. At the end of a block of 96 trials, the subject had a break of approximately $10 \mathrm{~min}$.

\section{RESULTS}

The relation between mean correct response latency and memory load, $\mathrm{M}$, is shown in Figure 2. Considering the $\mathrm{D}=1$ condition, the results show a significant linear relation (for positive responses, $\mathrm{F}=$ 51.6; for negative responses, $F=37.1$; df $=1,3$; $p<.01)$ but no significant quadratic component in either case. The best fitting straight lines for the positive and negative responses were approximately parallel, the ratio for the slopes of positive to negative responses being 1.05 .

For the $D=M$ condition, positive and negative responses clearly exhibit deviation from linearity, being concave upward. An analysis of variance for a

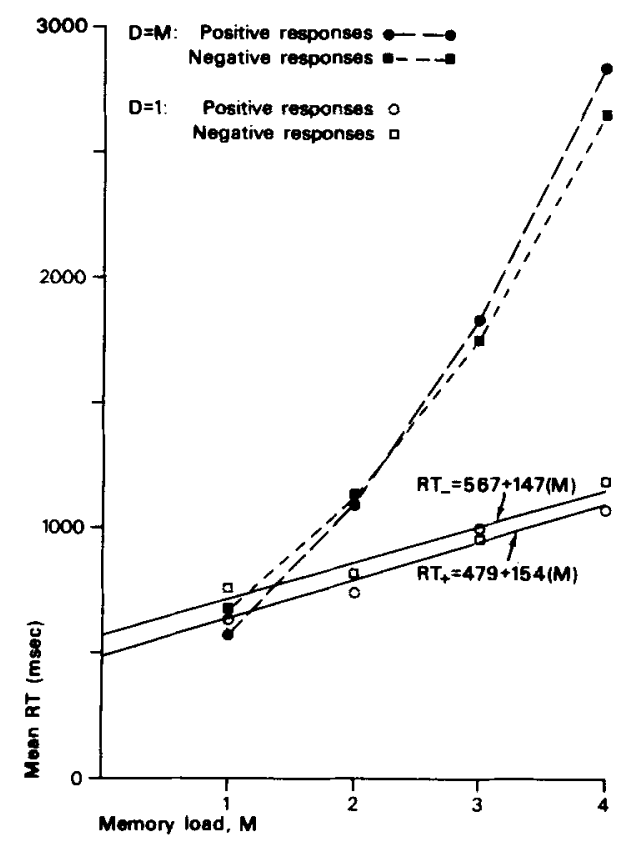

Figure 2. Relation between RT and $M$ for pooltive and negative responses under the $D=1$ and $D=M$ condition. The solid lines under the $D=1$ condition represent the best fitting straight lines to these data obtained from eight subjects. factorial design was performed for positive and negative responses separately, using data from individual subjects. A significant main effect of $M$ was found for both negative $(F=7.3 ; \mathrm{df}=3,21 ; \mathrm{p}<.01)$ and positive responses $(\mathrm{F}=13.3 ; \mathrm{df}=3,21$; $p<.01)$. Both positive and negative responses were tested for a quadratic component, which was found to be significant at the $5 \%(\mathrm{~F}=4.7 ; \mathrm{df}=1,21)$ and $1 \%$ ( $F=8.3 ; \mathrm{df}=1,21)$ levels, respectively. It is against these data that models of the processes underlying search tasks will be evaluated in the discussion.

The data for negative trials under the $D=M$ condition were further analyzed by plotting individual RT as a function of the position, $\mathrm{p}_{i}$ of the negative item, for each value of $M$. Examination of these individual data revealed that three of the eight subjects show clear serial position effects. These data are shown in Figure 3.

Subject 2 (Figure 3a) and Subject 4 (Figure 3c) showed a left-to-right scanning order, with RT fastest for $D=1$ increasing with increases in display load to $D=4$. Subject 3 (Figure $3 b$ ) appeared to scan outwards from a central position of the display, half the time in each direction. Thus it is likely that the overall lack of serial position effects arose from the use of different strategies across subjects. The lack of a serial position effect in an individual subject's data may be explained by a random order of search. The three subjects who did exhibit serial position effects all used their right hands to indicate a positive response. It may be noted that key-to-response assignment apparently has some strategic importance in uncovering self-terminating search processes. For example, Atkinson, Holmgren, and Juola (1969) reported an unpublished study in which differences in RT/display-size slopes were observed between subjects making a negative response on the left and on the right. These differences in slope have been revealed by key positioning and tend towards the predictions of a self-terminating model.

The data were subjected to an error analysis, and error rates across the two conditions were comparable $(5.4 \%$ for $\mathrm{D}=\mathrm{M}$ and $5.5 \%$ for $\mathrm{D}=1)$. Error data dependent on $M$ for the $D=M$ condition are shown in Figure 4. The major features of these data were the increase in the probability of a false negative response, i.e., a negative response when a positive response would be appropriate, with an increase in $M$ and the greater incidence of false negative over false positive responses for values of $M>1$.

\section{DISCUSSION}

\section{Analysis of the $D=1$ Condition}

In the simple character classification task, as we stated in the introduction, four models are usually considered. These arise from the combination of the two conceptual contrasts, serial or parallel and 

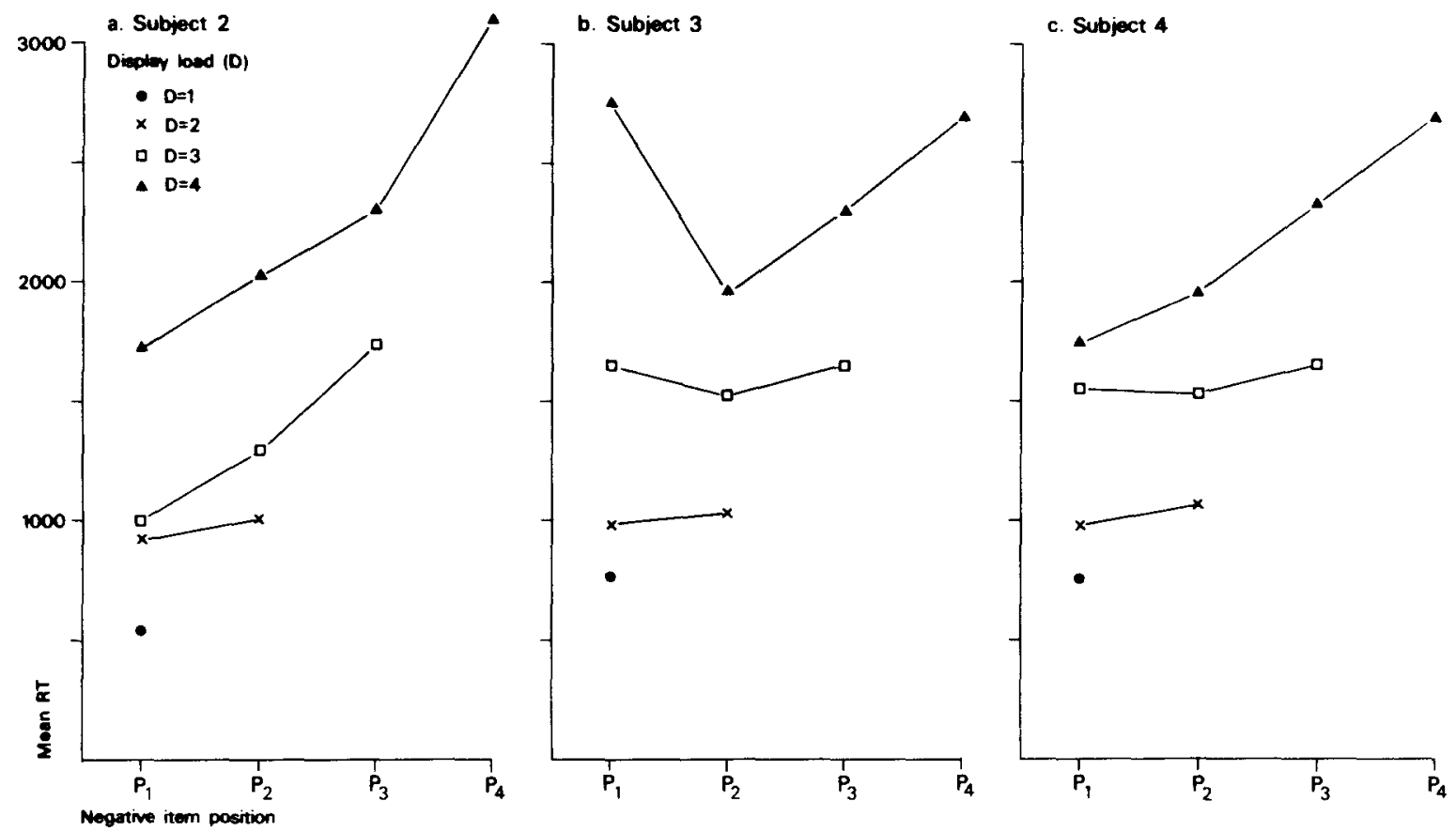

Figure 3. Relation between $R T$ and position, $p_{k}$, of the negative item under the $D=M$ condition for each value of $D$, for three individual subjects.

exhaustive or self-terminating. The linear relation between RT and $M$ observed in this experiment is congruent with other findings using a similar task. As pointed out earlier. no significant quadratic component was found. The existence of a negatively accelerated quadratic component would have indicated a logarithmic relation between $\mathrm{RT}$ and $\mathrm{M}$ which has been reported by some experimenters (Johnsen \& Briggs. 1973). The observed linearity together with the comparability of slopes for positive and negative responses have been interpreted as support for a serial exhaustive search of the memory set (Sternberg. 1966).

\section{Analysis of the $D=M$ Condition}

Extending the task by requiring classification of more than one character gives rise to a further set of models for this additional dimension. In order to consider models for how these characters are scanned, theorists have usually assumed display encoding and memory comparison do not overlap in time. If we make this assumption, no further elaboration of the models is required and we have the 16 models resulting from the combination of the 4 models of the simple character classification task and the 4 models for the additional dimension in the modified task. The serial exhaustive-memory/self-terminating-display search model proposed by Sternberg (Note 1) to explain his data and outlined in the introduction is an example of one of these models.

In order to simplify consideration of the models, we will first make the assumption that memory and display scan are serial. This can be justified in that a plot of RT vs. $M$ for the $D=1$ condition gave a linear function which Sternberg interpreted as support for a serial scan of memory. Similarly. Atkinson et al. (1969) interpreted their display scan study as consistent with a serial scan of display. These considerations eliminate all but the serial-memory/ serial-display models. While the linearity of the

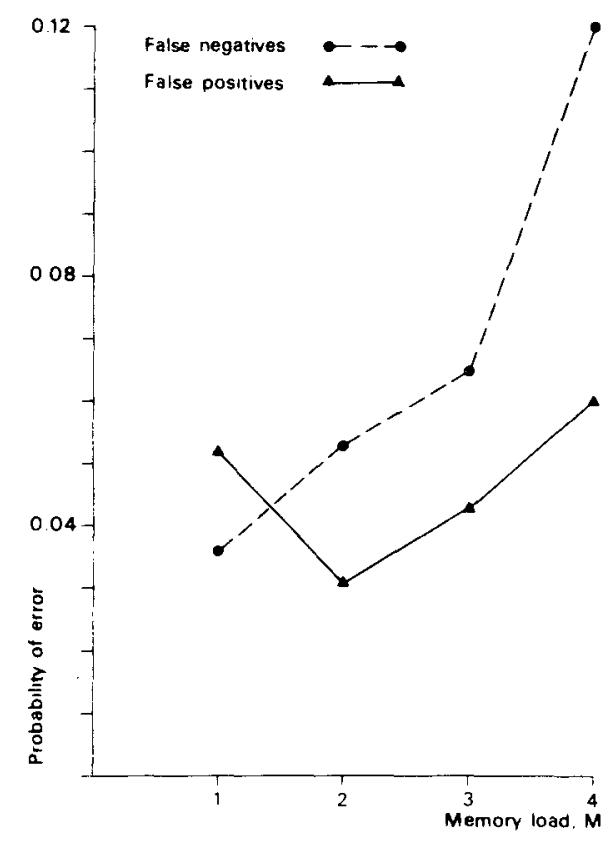

Figure 4. Probability of false ponitive and false negative responses as a function of memory load, $M$, under the $D=M$ condition. 
simple memory and display scan functions has been interpreted as support for a serial process, a parallel scanning model may also predict a linear increase in both positive and negative RT with the task variable (memory or display comparisons), as Atkinson et al. (1969) have shown. It should be noted, however, that the model outlined by Atkinson does require an exhaustive scan, and an exhaustive scan of a dimension does not permit any definitive conclusions about the serial or parallel nature of scanning processes for that dimension. For example, the data which Sternberg explained in terms of a serialexhaustive - memory/serial - self-terminating - display model can equally well be explained in terms of a parallel - exhaustive - memory/serial - self-terminatingdisplay model.

\section{Evaluation of Models for the $D=M$ Condition}

Initially, let $t_{1}$ be the time to encode an item from the display and $t_{2}$ be the time to compare an encoded representation against a memorized item.

Assuming serial interrogation of display (D) and memory (M), a general equation relating RT to $D$ and $M$ can be derived (Equation 1).

$$
R T=f_{1}[D]\left[t_{1}+f_{2}(M) t_{2}\right]+R,
$$

where $f_{1}(D)$ weights the number of items encoded from the display for a given value of $D, f_{2}(M)$ weights the number of comparisons of the encoded representation against the memorized items for a given value of $\mathrm{M}$, both of which depend on the type of response and exhaustive or self-terminating nature of processing for the dimension under consideration. $R$ represents the time required to produce a response.

In this experiment, the general equation can be expanded as a quadratic:

$$
R T=R+t_{1}\left[f_{1}(D)\right]+t_{2}\left[f_{1}(D) \cdot f_{2}(M)\right] .
$$

The functions for the conceptual contrast (exhaustive vs. self-terminating) are presented in Table 1 separately for display and memory and for response type.

It should be noted that predictions for display self-terminating models do not require qualification

Table 1

Functions for Display and Memory Conceptual Contrasts

\begin{tabular}{llcc}
\hline & & $\mathrm{f}_{1}(\mathrm{D})$ & $\mathrm{f}_{2}(\mathrm{M})$ \\
\hline \multirow{2}{*}{ Exhaustive } & Positive & $\mathrm{D}$ & $\mathrm{M}$ \\
& Negative & $\mathrm{D}$ & $\mathrm{M}$ \\
\hline & Positive & $\mathrm{D}$ & $\frac{\mathrm{M}+1}{2}$
\end{tabular}

Self-terminating

Negative $\quad \frac{D+1}{2} \quad \frac{M+1}{2}-\frac{M+1}{2\left(f_{1} D\right)}+\frac{M}{f_{1} D}$

Table 2

Predicted Relations Between the Linear $\left(t_{1}\right)$ and Quadratic $\left(t_{2}\right)$ Components for Positive $(+)$ and Negative $(-)$ Responses

Model

(Memory Search/

Display Search) Predicted Relations Between $t_{1}$ and $t_{2}$

$\begin{array}{llll}\begin{array}{l}\text { Exhaustive/ } \\ \text { Exhaustive }\end{array} & \mathrm{t}_{1+}=\mathrm{t}_{1-} & \mathrm{t}_{2+}=\mathrm{t}_{2-} & \\ \begin{array}{l}\text { Self-terminating/ } \\ \text { Exhaustive }\end{array} & & \mathrm{t}_{2+}=2 \mathrm{t}_{2-} & \mathrm{t}_{1-}>\mathrm{t}_{2-} \\ \begin{array}{l}\text { Exhaustive/ } \\ \text { Self-terminating }\end{array} & \mathrm{t}_{1+}<\mathrm{t}_{1-} & \mathrm{t}_{2+}=\mathrm{t}_{2-} & \mathrm{t}_{1+}>\mathrm{t}_{2+} \\ \begin{array}{l}\text { Self-terminating/ } \\ \text { Self-terminating }\end{array} & & \mathrm{t}_{2+}=\mathrm{t}_{2-} & \mathrm{t}_{1-}>\mathrm{t}_{2-}>\mathrm{t}_{2+} \\ & & & \mathrm{t}_{1-}>\mathrm{t}_{2-}\end{array}$

Note-These predictions are derived from models involving serial scanning of both memory and display items, in which display encoding time increases as a function of the number of items on display.

as to whether or not individual subjects show a defined or random order of search. When there is a defined order of search. the probability of encountering a decision item at the lirst to the M-th scanned position is the same since this is experinentally controlled. With a random order of search. the probability of encountering a decision item at the first to the M-th scanned position is again the same, if order of scan is independent of the decision item position.

Functions of the form given in Equation 2 were fitted using a polynomial regression technique. for positive and negative responses separately, giving values as follows:

Positive responses:

$$
\mathrm{RT}=314.25+140.15(\mathrm{M})+122.75\left(\mathrm{M}^{2}\right)
$$

Negative responses:

$$
\mathrm{RT}=459.00+111.20(\mathrm{M})+109.00\left(\mathrm{M}^{2}\right)
$$

Substituting into Equation 2 the expressions for D and $M$ as demanded by the exhaustive and self-terminating cases enables predictions concerning the relative magnitude of the linear and quadratic components to be derived. These are presented in Table 2.

These predictions are difficult to evaluate statistically given the Latin square design and the attempt to prove the null hypothesis in some cases. However, among the alternatives, one may suppose that the exhaustive-memory/exhaustive-display model is favored. Thus, $t_{1+}$ is approximately equal to $t_{1-}$ and $t_{2}+$ is approximately equal to $t_{2-}$. The exhaustive-exhaustive model is the only one to give these predictions, as indicated in Table 2. The time taken for comparing an encoded display item against 
a memorized representation $\left(t_{2}\right)$ takes 122.75 and $109.00 \mathrm{msec} /$ comparison for positive and negative responses. respectively. The time to encode display items $\left(t_{1}\right)$ takes the values 140.15 and $111.20 \mathrm{msec} /$ item for positive and negative responses.

It is not apparent why positive and negative probes require different amounts of time to encode, nor why there is a difference in comparison time. The comparison times could be made congruent by assuming that rechecking takes place on positive trials inflating the observed comparison time. The comparison times show marked discrepancies with the $\mathrm{D}=1$ condition. If, as has been found, memory and display comparison times are equal (Nickerson, 1966), we would expect the comparison times for the $\mathrm{D}=\mathrm{M}$ and $\mathrm{D}=1$ conditions to be the same. Comparison times for the $\mathrm{D}=1$ condition are 154 and $147 \mathrm{msec} /$ item, as indicated in Equations 5 and 6 . This prediction, therefore, is not fulfilled.

Positive responses for $\mathrm{D}=1$ condition:

$$
\mathrm{RT}=479+154(\mathrm{M})
$$

Negative responses for $\mathrm{D}=1$ condition:

$$
\mathrm{RT}=567+147(\mathrm{M})
$$

If the exhaustive-exhaustive model is favored, then it must be shown why some subjects exhibit serial position effects. One possibility is to attribute the effects to a compatibility artifact. For example, Holmgren (1974) entertained the hypothesis that when a decision item is detected on the left side of the display, a left-hand decision response is made faster than a right-hand one, with the reverse being true when the decision item is on the right of the display. If this were the case in the present task, apparent serial position effects might arise. However, these would be expected in different directions for the two key assignments.

Consideration of the error probabilities show's that. for all four models, the probability of false negative and false positive responses are given by the following expressions (assuming independent comparisons):

$$
\mathrm{P}(\text { false negative })=1-\left[1-\left(1-\mathrm{p}_{+}\right)^{\mathrm{M}-1} \mathrm{p}_{-}\right]^{\mathrm{M}}
$$

$\mathbf{P}($ false positive $)$

$$
=\left[1-\left(1-\mathrm{p}_{+}\right)^{\mathrm{M}-1} \mathrm{p}_{-}\right]^{\mathrm{M}-1}\left[1-\left(1-\mathrm{p}_{+}\right)^{\mathrm{M}}\right]
$$

where $M$ is the number of memory items; $p_{+}$is the probability of a false match when comparing two different items: $p_{-}$is the probability of a false mismatch when comparing two matching items.

These expressions are the same for the exhaustive and self-terminating cases, since the only difference between exhaustive and self-terminating processing is that in the former case processing continues after sufficient information for a response has been obtained. If $p_{+}=p_{-}$, then the error rate is the same for both response types, but if $p_{-}>p_{+}$, the predicted error probabilities show qualitative similarities to the data. The error data do not allow us to discriminate among the models.

The exhaustive-memory/exhaustive-display model has difficulties in dealing with serial position effects and differences in slope between response types and comparison of the slopes under the $D=M$ and $\mathrm{D}=1$ conditions. Final rejection of the model will have to await an experiment in which $\mathbf{D}>\mathbf{M}$, where the predicted differences in latencies permit more definitive conclusions.

Although the weight of the evidence against the exhaustive-exhaustive model is in no way conclusive, we extend our treatment by making the assumption, outlined in the introduction, that display encoding time is a constant. The implication of this is to make $f_{1}(D) t_{1}$ in Equation 2 a constant.

Making this assumption, the positive and negative response data for the $D=M$ condition were reexamined in terms of the number of comparisons demanded by the various models. The logic for this reanalysis is that in the simple character classification and display scan studies the task variables are memory and display comparisons, respectively. Data analysis for these tasks usually proceeds by plotting RT vs. $M$ or $D$ for positive and negative responses separately. The form and slope of the resulting plots are then compared with those predicted by the proposed models. In the present task, going from a memory and display load of $\mathbf{M}=\mathbf{N}$ to $\mathbf{M}=\mathbf{N}+\mathbf{1}$ clearly gives an additional display item as well as an additional memory item. Display and memory comparisons therefore interact multiplicatively in the models considered here but possibly with different weighting factors for the two response types, depending on whether the dimension under consideration is examined exhaustively or in self-terminating fashion. The predicted number of $D$ with $\mathbf{M}$ comparisons for each of the alternative models may be computed as the weighting factor for $t_{2}$, i.e., the value which is formed by the product of $f_{1}(D) \cdot f_{2}(M)$ in Equation 2 .

Table 3 presents latencies and the predicted number of comparisons for the various models. If the positive and negative response latencies are plotted against the hypothesized number of $D$ with $M$ comparisons. then all four models provide a satisfactory linear fit. This supports a serial process or a parallel model of the type presented by Atkinson et al. (1969). On the basis of the reanalysis of the data, the resulting functions for positive and negative responses should be parallel; however. in all cases, the slope for positive responses is significantly greater 
Table 3

Observed Latencies for Positive (+) and Negative (-) Responses for Each Value of Memory Load (M) Under the $D=M$ Condition and the Corresponding Predicted Number of Comparisons

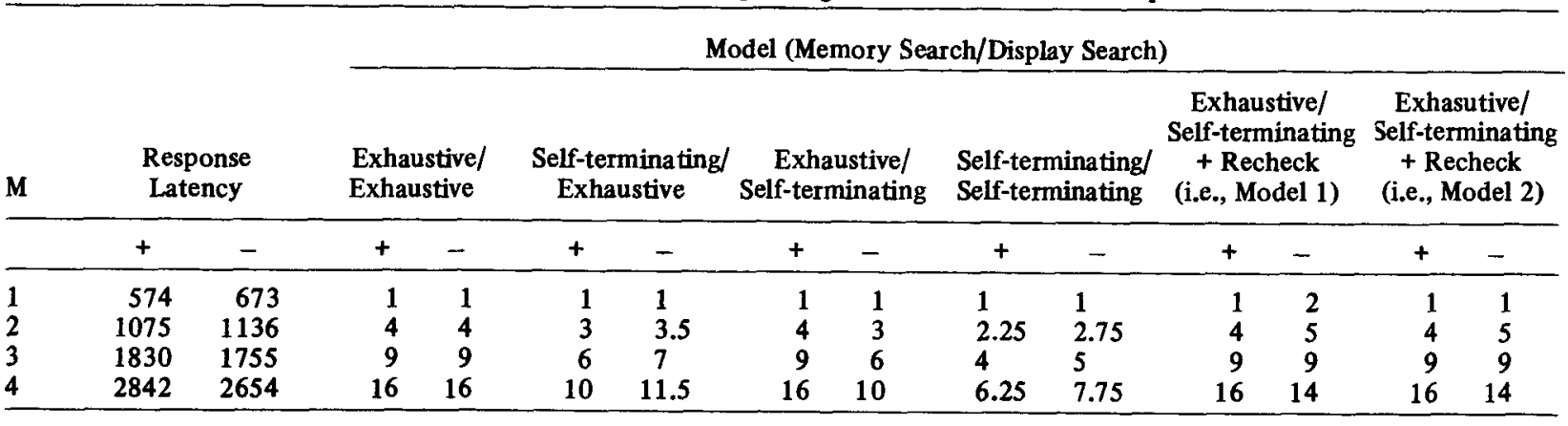

Note-These predictions are derived from models involving serial scanning of both memory and display items, in which display encoding time is assumed to be a constant.

than that for negative responses, as shown in Table 4. Thus, no model as it stands can adequately account for the obtained latency data.

The ability of the various models to explain the error and serial position.data is also presented in Table 4. As pointed out earlier, the error data can equally well be explained by any of the four models and do not permit discrimination among them. The serial position effects are not compatible with all of the four models but can be accommodated by the inclusion of additional assumptions. In the case of the exhaustive display models, it would be necessary to assume that the serial position effects are artifactual, and this artifact occur's for only one key assignment. For the self-terminating memory models, a random order of memory search would be required for those subjects not showing serial position effects.

All features of the data can be dealt with satisfactorily by both the exhaustive-memory/selfterminating-display and self-terminating-memory/ self-terminating-display models. Furthermore, an exhaustive-memory/self-terminating-display model can be made compatible with the observed differences in latency of positive and negative responses if a recheck is proposed on those trials on which a negative outcome ensues.

The notion of rechecking to confirm the negative set hypothesis prior to emitting a negative response was introduced by Briggs and Blaha (1969) for character classification and Krueger (1973) for "same""different" judgments. Our view of rechecking assumes that the subject performs a recheck only if his first scan indicates that a negative response is appropriate. The recheck takes the form of reaccessing the criterion set and performing an exhaustive search for the negative item.

In considering the $R T$ data under the $D=M$ condition in terms of the predicted number of comparisons, for $D=M=1$ there are two alternative models depending on whether or not the rechecking process obtains. In this condition, we have a situation comparable with Sternberg's initial character classification studies and also to Atkinson's display scan studies, both of which revealed an exhaustive scan which requires no recourse to rechecking. Besides such empirical considerations as to the absence of a recheck, it is easy to conceptualize an underlying process. When $D=M>1$, the subject registers two kinds of information when he scans an item, that is position and identity information.

In contrast, when $D=M=1$, the subject has only one kind of information, i.e., identity, and an exhaustive scan might be regarded as sufficient without a recheck. The models where rechecking is (Model 1) and is not (Model 2) involved for the case

Table 4

Models in Which Display Encoding Time is a Constant and Their Ability to Explain the Major Experimental Findings

\section{Major Experimental Findings}

\begin{tabular}{|c|c|c|c|c|c|}
\hline $\begin{array}{c}\text { Model } \\
\text { (Memory Search/ } \\
\text { Display Search) }\end{array}$ & $\begin{array}{r}\text { Significanc } \\
\text { Slopes for } \\
\text { Respons } \\
\text { Two }\end{array}$ & $\begin{array}{l}\text { erence in } \\
\text { Negative } \\
\text { t Test, } \\
\text { ) }\end{array}$ & $\begin{array}{l}\text { Error } \\
\text { Data } \\
\text { (Figure 4) } \\
\end{array}$ & $\begin{array}{c}\text { Display } \\
\text { Position } \\
\text { Effects }\end{array}$ & $\begin{array}{c}\text { Memory } \\
\text { Position } \\
\text { Effects } \\
\end{array}$ \\
\hline $\begin{array}{l}\text { ve/Exhaustive } \\
\text { ive/Self-terminating } \\
\text { inating/Exhaustive } \\
\text { inating/Self-terminating }\end{array}$ & $\begin{array}{l}t=2.328 \\
t=4.145 \\
t=5.210 \\
t=7.164\end{array}$ & $\begin{array}{l}p<.05 \\
p<.05 \\
p<.01 \\
p<.01\end{array}$ & $\begin{array}{l}t \\
t \\
t \\
t\end{array}$ & $\begin{array}{l}x(\dagger) \\
\dagger \\
x(\dagger) \\
\dagger\end{array}$ & $\begin{array}{l}\dagger \\
\dagger \\
\mathbf{X}(\dagger) \\
\mathbf{X}(\dagger)\end{array}$ \\
\hline
\end{tabular}

Self-terminating/Self-terminating

Note- $t$ and $X$ refer to the model's success or failure, respectively, to explain the observed findings; ( $t$ ) means the model can explain the findings if additional assumptions are made as outlined in the Discussion. 
$\mathrm{D}=\mathrm{M}=1$ were both fitted to the data, and the equations for negative responses of the best fitting straight lines were, respectively:

$$
\begin{aligned}
& \text { Model 1: RT }=319+165 \mathrm{C} \\
& \text { Model 2: RT }=443+153 \mathrm{C}
\end{aligned}
$$

where $C$ refers to the number of scanning comparisons executed. This compares with the following equation of the line fitted to positive responses:

$$
\mathrm{RT}=461+150 \mathrm{C}
$$

All three equations were significantly linear beyond the $0.1 \%$ level $(\mathrm{F}=1205,217$, and 1635 , respectively; $\mathrm{df}=1,3)$. No significant differences in slope were found between either of the two lines fitted to the negative responses obtained under the $M=1$ condition, and the slope of the line fitted to positive responses. There is a significant difference in intercept between the lines fitted to negative responses under Model 1 and to positive responses $(t=3.994$; df $=7 ; p<.05$, one-tailed test), but no significant difference emerges when considering Model 2.

Comparison of the slope constants under the $\mathrm{D}=\mathrm{M}$ condition for the proposed model and for the best-fitting lines for the $D=1$ condition revealed that they are remarkably similar. (For negative responses, the difference is $6 \mathrm{msec}$, and for positive responses it is $4 \mathrm{msec}$ ). Of all the models considered, the exhaustive-memory/self-terminating-display model with rechecking on negative trials when $M>1$ ofters the most satisfactory account of the evidence. This model predicts a common slope for negative and positive responses when plotted against the number of comparisons involved. The slope constants for the $\mathrm{D}=\mathrm{M}$ condition under this model and for the $\mathrm{D}=1$ condition are very similar. In addition, the model provides an explanation of the display serial position effects. In contrast, the self-terminatingmemory/self-terminating-display model would require the additional assumption of rechecking on both positive and negative trials to explain the latency data and seems implausible.

Under the $\mathrm{D}=\mathrm{M}$ condition, a feature of the data was the upward concavity evident in the relation between RT and $M$ for both positive and negative responses. In order to confirm that this concavity had been removed, a polynomial regression was performed on positive and negative responses separately after standardization of the abscissa in terms of the number of scanning comparisons predicted by the modified exhaustive-memory/self-terminating-display model. For neither positive nor negative responses did the quadratic component which reflects such concavity reach significance. Thus, the standardization was successful in removing the upward concavity.
Implications of the Exhaustive-memory/SelfTerminating-Display Scan Model for Character Classification and "Same"..Different" Judgment Tasks

Character classification. It was noted in the introduction that a common feature of studies in which both number of items in memory and number of items on the display are varied is that negative latencies are not accurately predicted by a simple exhaustive-memory/self-terminating-display model. The inclusion of an intuitively plausible rechecking scheme allowed accurate quantitative predictions of response latencies to be made.

It was suggested that the lack of display position effects in other studies might arise from a failure to consider the relation of negative and positive responses to the hands producing such responses and to different strategies used by subjects.

"Same"-"different" judgments of letter strings. In this study, a positive response is equivalent to a "same" response of the "same"-"different" judgment task. One common finding in such studies is that "same" responses are faster than "different" responses. Our analysis permits the dissociation between comparison and other stages involved in these tasks. Our model suggests that there is a lengthening of comparison times caused by rechecking. ${ }^{1}$

One feature which puzzled Bamber was the upward concavity of "same" responses in his data. For different responses, he proposed a self-terminating display model. Our model proposes that the subject deals with one item at a time from the display and performs an exhaustive search against memory and terminates as soon as a display item is found to be not a member of the CS. This is successful in predicting the upward concavity observed here and in Bamber's experiments.

The model's success in predicting the upward concavity would seem to highlight the similarity between our task and that of Bamber, but how might we generalize the model to a situation where position is important as in Bamber's experiment? We can take a lead from an experiment by Bamber and Paine (1973). They focused their attention on certain mechanisms by which information is retrieved from letter strings. Two types of information are available - position and identity. Bamber and Paine explicitly made the assumption that the CS and TS are both encoded in the form of a.letter and position tag. If we make this assumption about the encoding of items in "same"-"different" judgments of letter strings, then our model is directly applicable. The scan would then be an exhaustive search of position and identity pairs of the CS vs. each position and identity pair of the TS until a decision ensued. However, it does not even appear to be necessary to make this additional assumption. While different 
items could be different with respect to just position or just identity, the former has usually not been considered.

\section{REFERENCE NOTE}

1. S. Sternberg. Scanning a persisting visual image versus a memorised list. Paper presented at the Annual Meeting of The Eastern Psychological Association. 1967.

\section{REFERENCES}

Atrinson, R. C., Holmgren, J. E., \& Juola, J. F. Processing time as influenced by the number of elements in a visual display. Perception \& Psychophysics, 1969, 6, 321-326.

BAMBER, D. Reaction times and error rates for "same". "different" judgments of multidimensional stimuli. Perception \& Psychophysics, 1969, 6. 169-174.

BAMBER, D. Reaction times and error rates for judging nominal identity of letter strings. Perception \& Psychophysics. 1972. 12, 321-326.

BAMBER, D., \& PAINE, S. Information retrieval processes in "same"." "different" judgements of letter strings. In S. Kornblum (Ed.). Attention and performance IV. New York: Academic Press, 1973.

Briggs. G. E., \& Blaha, J. Memory retrieval and central comparison times in information processing. Journal of Experimental Psychology, 1969, 79, 395-402.

Holmgren, J. E. Visual search in a forced-choice paradigm. Perception \& Psychophysics, 1974, 16, 253-258.

Johnsen, A. M., \& Briggs, G. E. On the locus of display load effects in choice reactions. Joumal of Experimental Psychology, 1973, 99, 266-271.
KRUEGER, L. E. Effect of irrelevant surrounding material on speed of "same"-"different" judgments of two adjacent letters. Journal of Experimental Psychology, 1973, 98, 252-259.

Nickerson, R. S. Response times with a memory-dependent decision task. Journal of Experimental Psychology, 1966, 72 , $761-769$.

Nickerson, R. S. Binary-classification reaction time: Review of some studies of human information-processing capabilities. Psychonomic Monograph Supplements, 1972, 4, Whole No. 65.

Rosenbaum, D. A. Rule use in character classification: Are serial parallel processing discrete? Memory and Cognition, 1974, 2, 249-254.

STERNBERG, S. High-speed scanning in human memory. Science, $1966,143,652-654$.

\section{NOTE}

1. In the present experiment, for the $D=M$ condition, the proportion of CS-TS pairs with respect to position and identity decreases as $M$ increases. It might be argued that this could account for the faster positive responses. Our on-line analysis of the data does not allow us to test this hypothesis. However, one could question this explanation in that for the $D=1$ condition the difference between positive and negative responses remains approximately constant despite the fact that identity between the CS and TS occurs only when $M=1$.

(Received for publication April 18, 1975; revision received July 28,1975 .) 